\title{
The success of periclavicular brachial plexus block
}

\author{
Jeong Uk Han \\ Department of Anesthesiology and Pain Medicine, Inha University Hospital, Incheon, Korea
}

There were two papers published on brachial plexus block (BPB) this month. One was on the difference in outcome between infra and supra-clavicular approach using nerve stimulator when blocking the upper arm from the elbow joint down [1], and the other paper compared the outcome between ultra-sonography guided supra-clavicular approach with direct visualization of the brachial plexus to cases where visualization was not achieved [2]. Both approach methods showed high success rates in achieving nerve block of the upper arm but incidence of Horner's syndrome and pneumothorax was lower in the infra-clavicular block group in the fist study. The second study showed that even when direct visualization was not achieved or was vague, injection around the subclavian artery, though the success rate was a bit low, was adequate enough to achieve nerve block and there was no significant difference in the complication rate, not to mention that there was no case of pneumothorax.

For a successful brachial plexus block to be achieved there are many factors to be considered, one particular factor is the precise targeting of the nerve itself and there have been various methods to do so. Such as inducing parethesia through direct nerve contact, direct injection into the peri-arterial sheath via arterial puncture, identifying sensory and motor response using nerve stimulators, and the more recently acclaimed visualization of surrounding anatomical structures using ultrasonography resulting in higher success rates in achieving brachial plexus block. There are a number of recent studies on BPB comparing the effectiveness of nerve block achieved by the combined use of nerve stimulator and ultra-sonography, and the independent use of each method separately which show diverse results depending on the researcher, but the use of ultra-sonography guided BPB prevails overwhelmingly in success rate, complication rate, shorter duration of application and patient satisfaction [3-7].

The anatomical pathway to reach the brachial plexus is diverse. Since the first report on the variation of the brachial plexus over a 100 years ago to more recent cadaver autopsies showing cord level variations in up to $12.8 \%$ of cases [8], acknowledge the diverse range of variations associated with the brachial plexus which can not only play a definite role in the failure of BPB, but can also increase the rate of complications due to multiple punctures when using the nerve stimulator, which in turn makes the usefulness of ultra-sonography more valid. Nerve block performed under ultra-sonography does not come without a financial burden, but there has been a report acknowledging that the use of ultra-sonography financially overpasses the nerve stimulator in the long run [9].

In conclusion, even though the introduction of the nerve stimulator and nerve block needle immensely advanced BPB, it cannot be compared to the advantages brought about by ultrasonography. Furthermore, ultra-sonography is continuously developing into 3-dimensional and 4-dimensional even at this moment. Nevertheless, there are still many hospitals without even the most basic ultra-sonograhic equipment and I hope that in the very near future all medical facilities become equipped with ultra-sonography.

\section{References}

1. Yang CW, Kwon HU, Cho CK, Sung MJ, Kang PS, Park ES, et al. A comparison of infraclavicular and supraclavicular approaches to the brachial plexus using neurostimulation. Korean J Anesthesiol

Corresponding author: Jeong Uk Han, M.D., Ph.D., Department of Anesthesiology and Pain Medicine, Inha University Hospital, 7-206, 3-Ga, Sinheung-dong, Jung-gu, Incheon 400-711, Korea. Tel: 82-32-890-3969, Fax: 82-32-881-2476, E-mail: jwhanan@inha.ac.kr (c) This is an open-access article distributed under the terms of the Creative Commons Attribution Non-Commercial License (http:// creativecommons.org/licenses/by-nc/3.0/), which permits unrestricted non-commercial use, distribution, and reproduction in any medium, provided the original work is properly cited. 
2010; 58: 260-6

2. Jeon DG, Kim WI. Cases series: ultrasound-guided supraclavicular block in 105 patients. Korean J Anesthesiol 2010; 58: 249-55.

3. Orebaugh SL, Williams BA, Vallejo M, Kentor ML. Adverse outcomes associated with stimulator-based peripheral nerve blocks with versus without ultrasound visualization. Reg Anesth Pain Med 2009; 34: 251-5.

4. Klaastad Ø, Sauter AR, Dodgson MS. Brachial plexus block with or without ultrasound guidance. Curr Opin Anaesthesiol 2009; 22: 655-60.

5. Han JU, Jung JK, Lim HK, Lee JH, Seo CH, Shinn HK. Usefulness of ultrasound-guided infraclavicular brachial plexus block: a comparison with nerve stimulation method. Korean J Anesthesiol
2008; 55: 436-40.

6. Sauter AR, Dodgson MS, Stubhaug A, Halstensen AM, Klaastad Ø. Electrical lateral sagittal infraclavicular blocks: a randomized, controlled, observer-blinded, comparative study. Anesth Analg 2008; 106: 1910-5.

7. Michael LB, Brian DS, John DG. Use of a nerve stimulator does not improve the efficacy of ultrasound-guided supraclavicular nerve blocks. J Clin Anesth 2006; 18: 580-4.

8. Pandey SK, Shukla VK. Anatomical variations of the cords of brachial plexus and the median nerve. Clin Anat 2007; 20: 150-6.

9. Sandhu NS, Sidhu DS, Capan LM. The cost comparison of infraclavicular brachial plexus block by nerve stimulator and ultrasound guidance. Anesth Analg 2004; 98: 267-8. 